\title{
Pascal BRISSETTE
}

\section{Postures et mises en scène d'auteurs}

Ce nouvel ouvrage de Jérôme Meizoz s'inscrit pleinement dans le sillon ouvert par L'Âge du roman parlant (2001), Le gueux philosophe (2003) et L'ail sociologue et la littérature (2004). Il s'agit bien encore ici pour l'universitaire, écrivain et critique littéraire suisse de combiner les acquis de la sociologie de la littérature et de la poétique afin de saisir dans l'analyse du texte (non fictionnel ou fictionnel) son double caractère individuel et collectif. À l'instar d'un Alain Viala ou d'un Dominique Maingueneau, mais aussi des sociocritiques dont il se réclame moins ouvertement (le mot apparaît p. 152), Meizoz veut renvoyer dos à dos ceux pour qui la littérature relève de la création pure, expression absolument singulière d'un être unique, ou n'est, à l'opposé, qu'un simple produit du social ou un effet de champ. C'est à cette fin qu'il approfondit le concept de posture, celui-ci appelant à la fois la mobilisation de données sur l'état du champ et la trajectoire de l'auteur ainsi que l'analyse de processus textuels et permettant de combiner approche externe et approche interne.

Le chapitre premier du volume, auquel Meizoz a donné la forme ingénieuse d'un dialogue entre un "Curieux» et un "Chercheur», explore le concept de posture, qui est emprunté à Viala et que l'auteur avait déjà défini et illustré (notamment dans les essais de L'œil sociologue). Le concept s'offre au chercheur comme un outil visant à étudier les différentes manières dont les écrivains fabriquent, sans toujours en avoir conscience, leur image publique. Cette image, cette identité littéraire permet essentiellement aux écrivains d'occuper de façon singulière une position dans le champ littéraire. Car il y a différentes manières de négocier ou de renégocier sa place sur la scène littéraire et la posture, en orientant le regard de l'analyste sur les œuvres, les paratextes et les conduites non verbales de l'écrivain, met l'accent sur les différences et les différends qui se manifestent entre les agents qui peuvent occuper des positions aussi bien rapprochées qu'éloignées dans le champ littéraire. L'intérêt de ce chapitre, par 
rapport aux précédents essais de Jérôme Meizoz sur la posture, est d'abord d'insister fortement sur le caractère relationnel de l'analyse impliquée par ce concept. Une posture n'existe pas seule, pas plus qu'on ne peut concevoir la position d'un seul agent dans un champ littéraire sans se questionner parallèlement ou préalablement sur l'état de ce champ et l'espace des possibles. Une posture doit être analysée en relation avec les autres postures affichées par les principaux agents dans le même état du champ. Ces questions de "mise en relation» s'étendent également aux états antérieurs du champ littéraire, puisque celui-ci possède sa mémoire propre, «regorge de récits fondateurs, de biographies exemplaires » (p. 25) qui offrent aux écrivains quantité de matériaux symboliques qu'ils réutilisent dans la fabrication de leur posture propre. Une posture ne se construit pas de toutes pièces : elle se «bricole» en utilisant toutes sortes de matériaux (références, modèles, exempla, mythes) qui ont valeur de «référence » (Fernand Dumont, Genèse de la société québécoise) pour une communauté donnée.

Une autre question judicieusement soulevée par Jérôme Meizoz dans ce chapitre est celle de la conscience des écrivains et de la part de roublardise généralement associée aux questions de stratégies et de postures littéraires. Meizoz explique que, depuis l'émergence des genres comme l'autobiographie à la fin du XVIII siècle, et de plus en plus avec l'apparition dans les années 1830 du journal moderne, l'écrivain est sommé de présenter au public une image de lui-même qui puisse aider le lecteur à le situer sur l'échiquier littéraire. Or la posture, souligne Meizoz, n'est pas seulement le propre d'écrivains particulièrement conscients des enjeux promotionnels liés à la médiatisation de leur personne; elle est en fait "quelque chose de commun à tous les écrivains (et à tous les artistes en général), attaché à leur statut même: une façon de faire face [...] aux avantages et désavantages de la position qu'on occupe dans le "jeu" littéraire ou plus généralement artistique » (p. 20). Il convient donc de différencier la posture de la «pose » et de comprendre que la première ne peut être réduite à un artifice, même si, parce qu'elle signale la singularité d'un écrivain donné dans une position donnée, son étude est essentielle à l'analyse d'une stratégie littéraire particulière. 
Le corpus «naturel» d'analyse de la posture, comme l'a montré Meizoz dans ses précédents essais, est formé des correspondances, entretiens, journaux intimes, mémoires, autobiographies, «carnets de bord ", témoignages et de tous les textes par le biais desquels l'écrivain prend « position» sur la place publique (discours, manifeste, pamphlet, etc.) et où l'instance énonciative peut être associée directement à la figure de l'auteur. Mais que dire des œuvres de fiction et de leur rôle dans la fabrication de la posture littéraire? Le concept de posture peutil aider à mieux les lire et à déployer leur socialité? Meizoz répond en deux temps. D'une part, avance-t-il, on ne peut ramener la posture d'un auteur à celle d'un personnage parce que le premier s'est généralement «diffracté [en plusieurs personnages], usant de contrepersonnages, de doubles, d'opposants, etc.» (p. 28) Meizoz admet cependant que, dans le cas de certains écrivains, les œuvres de fiction ont fortement contribué à modeler leur identité littéraire, les lecteurs ayant fait de leurs romans ou poèmes une lecture biographique l'exemple de Rousseau en Saint-Preux est cité avec raison et on pourrait ajouter, entre autres exemples, celui de Hugo/Olympio. Mais ce n'est pas la voie que veut explorer Meizoz, peut-être moins à l'aise avec les questions de réception (que faire d'un public qui ignore ou veut ignorer que le « héros du roman » n'est pas l'auteur du livre?) et d'imaginaire (pourquoi le public n'utiliserait pas aussi, pour recréer une image de l'auteur, les matériaux symboliques mis à sa disposition par l'œuvre de fiction?) qu'avec les méthodes d'analyse du discours, qu'il maitrise parfaitement. C'est ce qui l'incite sans doute à répondre, dans un deuxième temps, que le ton (humour, ironie, pudeur, arrogance) ou le rythme narratif, souvent associés au style de l'écrivain, à sa "manière », peut servir sans aucun doute d'indicateur postural (p. 29). Seuls quelques éléments des œuvres de fiction - ceux qui sautent peut-être moins vite aux yeux du lecteur — pourraient être ainsi mis à contribution dans l'analyse de la fabrication posturale.

L'analyse posturale présuppose l'existence forte de l'image de l'auteur ainsi qu'un public intéressé par les faits et gestes du «créateur». Meizoz revient donc, dans le deuxième chapitre de l'ouvrage, sur les fortunes et infortunes de cette notion d'auteur aux $\mathrm{XIX}^{\mathrm{e}}$ et $\mathrm{XX}^{\mathrm{e}}$ siècles. Il y rappelle que la figure de l'auteur qui nous est si familière 
apparait dans la deuxième moitié du XVIII ${ }^{\mathrm{e}}$ siècle à la suite d'une augmentation significative du public lecteur, du prestige croissant de la fonction de l'écrivain et du culte que l'on voue, à la veille de la Révolution et au cours des années 1790, aux Voltaire et aux Rousseau, héros de la Révolution. La méthode biographique d'un Sainte-Beuve, mais encore et surtout le spectaculaire développement de la presse au $\mathrm{XIX}^{\mathrm{e}}$ siècle et l'attention qu'elle accorde à la personne de l'écrivain renforcent cette «demande biographique » (p. 35) et suscitent chez les hommes de plume la nécessité de se singulariser au sein d'un monde où le travail est, paradoxalement, de plus en plus collectif (naissance de l'éditeur, importance du critique littéraire, etc.). Au $\mathrm{XX}^{\mathrm{e}}$ siècle, une série d'auteurs (après Mallarmé, Proust et Valéry) et de courants critiques (du formalisme russe à la Nouvelle Critique) occultent le «moi social» de l'écrivain au profit du texte et de ses mécanismes propres. Le chapitre se termine sur une judicieuse précision qui ouvre la voie aux analyses des chapitres ultérieurs: si l'analyse posturale est historiquement rendue possible par la transformation du statut de l'auteur dans la deuxième moitié du XVIII ${ }^{\mathrm{e}}$ siècle, ce n'est pas ce statut qui l'intéresse au premier chef, non plus que les données sociologiques concrètes (comme les revenus du producteur). L'analyse posturale travaille essentiellement avec des « représentations de l'auteur, pensées en relation avec sa position dans le champ littéraire»(p. 45). Elle se rattache ainsi à la sociologie du champ, mais s'en distingue par son objet et ses fins.

Le troisième chapitre propose une lecture intertextuelle des motifs de la place et de l'ambition chez Rousseau (Confessions) et Stendhal (Le Rouge et le Noir). L'évolution de ces motifs est mise en rapport avec l'avènement de ce que Nelly Wolf appelle le «roman de la démocratie », c'est-à-dire le roman de l'ère bourgeoise, qui met en intrigue la valorisation de «l'ambition» (p. 47-48). Cet essai est peutêtre celui qui laisse le plus de questions en suspens dans l'ouvrage. L'intérêt réel de l'analyse proposée n'est certes pas à remettre en question, non plus que celui des conclusions - «[dans] Le Ronge et le Noir, l'épopée dégradée de l'amour porte dans son sillage les comédies du parvenir comme le bovarysme naissant» (p. 63) —, mais après les deux premiers chapitres théoriques, le lecteur pouvait s'attendre à voir 
se déployer une analyse qui tienne davantage compte des multiples aspects que le "Chercheur» avait expliqués au «Curieux» (chap. ${ }^{\text {en }}$ ). Or, étrangement, cette " posture démocratique moderne » que Meizoz reconstruit dans ce troisième chapitre à partir des Confessions et de Le Rouge et le Noir n'est complétée par aucune analyse de trajectoire ni ramenée à aucune position particulière, ni celle de Rousseau, ni celle de Stendhal. Comme si la posture avait ici une existence littéraire propre et ne s'intégrait à aucune stratégie particulière. On pouvait aussi s'attendre à ce que Meizoz tienne compte de «l'espace des positions littéraires du moment» (p. 21), c'est-à-dire des autres postures - les plus visibles — en regard desquelles celle qui est décrite dans Le Rouge et le Noir prend son sens. Or ce n'est pas le cas, et en dépit de la finesse déployée dans la lecture du roman stendhalien, cet essai parait un peu incomplet en regard des attentes suscitées par les deux chapitres d'ouverture.

Les chapitres suivants, tirés pour la plupart de communications ou d'articles parus antérieurement, peuvent parfois paraittre également incomplets, mais comme ils portent sur une même période historique (l'entre-deux-guerres) et sur un groupe d'auteurs bien précis (les tenants du «roman parlant» que connait bien Meizoz), ils se complètent et montrent les différentes manières dont on peut faire «travailler» le concept de posture. Tel chapitre (le cinquième) étudie les postures d'écrivains ayant pour trait commun l'authenticité et montre que la posture sert non seulement à distinguer les écrivains occupant des positions très éloignées dans le champ littéraire, mais aussi les agents qui défendent des positions similaires; tel autre chapitre (le septième) met en lumière l'utilisation que les écrivains peuvent faire de la biographie, tel Céline proposant avec son Semmelweis un modèle légitimant à l'avance ses propres écarts (le génie étant forcément incompris). Mais les deux analyses les plus fortes du volume sont proposées plus loin (chapitres VIII et IX).

Le chapitre VIII s'intéresse à la nouvelle «Venise » de Blaise Cendrars, où l'auteur de Bourlinguer (1948) met en scène un double écrivain (Manuci) qui «illustre [la] conception de la littérature "vivante" [de Cendrars]» (p. 126). Meizoz montre, par une analyse discursive, que 
"Venise» repose tout entière sur une série d'oppositions (écrivain / philologue; subversion / maitrise savante; authenticité /fausseté; mouvement / arrêt; vie / mort; oralité / écrit) qui valorisent la posture du bourlingueur, dont Manuci offre le modèle dans la nouvelle, véritable créateur seul capable de donner un souffle à la langue. Meizoz montre également que cette nouvelle ne prend tout son sens qu'en regard de la position qu'entend occuper Cendrars dans le champ littéraire français de l'après-guerre, en face d'un Sartre implicitement dévalorisé par le récit: "ainsi la posture du bourlingueur "pas très fort sur la grammaire" apparait-elle comme un pied-de-nez très contemporain à toute conception professorale de la littérature » (p. 150).

Le chapitre IX s'intéresse pour sa part à une querelle qui eut cours au sein de La Nouvelle Revue Française en juillet 1933 et qui opposa, d'un côté, André Gide et, de l'autre, Jean Paulhan et Charles-Albert Cingria. Ce dernier avait soumis à La NRF une note de lecture de L'Histoire de la révolution russe de Trotsky. Cingria proposa un compte rendu qui, parce qu'il osait poser un jugement de valeur strictement littéraire sur un ouvrage d'histoire, poussait la logique autonomiste de la littérature à sa limite. Et peut-être au-delà, si on s'en rapporte à Gide qui qualifia Cingria de «bouffon» et ne lui reconnut pas le droit de porter un tel regard, purement esthétique, sur l'ouvrage de Trotsky. L'intérêt de cet essai de Meizoz ne tient pas seulement à l'excellente analyse discursive qui est faite des textes de la querelle, il tient encore et surtout à l'attention qui est portée au «genre » comme catégorie de pensée et, en particulier, à ce genre réputé mineur qu'est la «note de lecture », mais qui est pourtant central au sein d'une revue comme La N.R.F. Comme le montre Meizoz en fin d'analyse, cette querelle renforce la posture de Cingria en bouffon des lettres et contribue à le mettre en évidence par rapport à «toutes les mises en scène sérieuses de l'autorité d'écrivain au nom d'idées morales ou politiques étrangères à la seule littérature, qu'il s'agisse des professions de foi marxistes des surréalistes, du moralisme littéraire dicté aux écrivains catholiques par le Syllabus (1864) ou du militantisme communisant d'André Gide » (p. 171).

Pour finir, diverses remarques pourraient être faites au sujet du travail éditorial. On trouve encore quelques coquilles, les répétitions ne sont 
pas rares (les catégories du prêtre et du prophète chez Weber sont expliquées à deux reprises et dans deux chapitres qui se suivent [le huitième et le neuvième]) et l'organisation des chapitres prête un peu à discussion (on aurait souhaité lire l'excellent chapitre VIII immédiatement après l'entrée en matière théorique du début, à la place du chapitre III actuel). Mais ce sont là des vétilles en regard des apports théoriques et critiques du volume. Le concept de posture que développe plus systématiquement ici Jérôme Meizoz permet un dialogue entre les approches «externes» du fait littéraire (sociologie du champ, des institutions, des réseaux, etc.) et les approches davantage centrées sur l'analyse interne et la socialité des textes (sociocritique, sociopoétique). Les lectures attentives que propose Meizoz, l'importance qu'il accorde au travail du discours, aux inflexions textuelles, à l'institution du genre, ne sont pas chose fréquente dans les études sociologiques et elles doivent être soulignées - et appréciées à leur juste mesure. Les limites de l'ouvrage sont pour l'essentiel attribuables à l'ambition même du programme de recherche ouvert par l'auteur: analyse du champ littéraire et des relations entre les diverses postures tenues à un moment précis de son développement, analyse de textes (fictionnels et non fictionnels) pour retracer l'élaboration d'une posture particulière, analyse des matériaux symboliques qu'elle réactive, lecture de texte attentive au travail sémiotique, etc. On voit mal comment un chercheur peut accomplir seul un tel travail et on ne peut qu'appeler de nos vœux la formation d'une équipe de recherche qui se donnerait pour mandat de tirer simultanément tous les «fils» de cette notion complexe et féconde. C'est là, nous l'espérons, la prochaine tâche que se donnera l'auteur de ce stimulant ouvrage, dont on ne saurait trop recommander la lecture.

Référence : Jérôme Meizoz, Postures littéraires. Mises en scène modernes de l'auteur. Essai, Genève, Slatkine Érudition, 2007, $204 \mathrm{p}$. 